\title{
DUA HASIL OPTIMAL DALAM PENYELESAIAN PERSOALAN TRANSPORTASI DENGAN ASSIGNMENT METHOD, VAM AND MODI, NORTHWEST CORNER AND STEPPING-STONE
}

\section{Rudy Santosa Sudirga}

Email: rudysudirga@yahoo.com

\section{Penulis}

Rudy Santosa Sudirga adalah pengajar di Universitas Bunda Mulia dengan peminatan dalam bidang manajemen operasi, manajemen sains dan manajemen pemasaran.

\section{Abstrak}

The famous method to determine and solve transportation problem is the transportation model and the assignment model. We see how to develop an initial solution to the transportation problem with VAM (Vogel's Approximation Method) and MODI (Modified Distribution) and Northwest Corner rule and the Stepping-Stone method. VAM is not quite as simple as the Northwest Corner approach, but it facilitates a very good initial solution, as a matter of fact, one that is often the optimal solution. The Assignment method, which is simple and faster to solve the transportation problem by reducing the numbers (cost) in the table/tableau until a series of zeros is found, or zero opportunity costs, which means that we will reach the optimal cost allocations. Once we have reached the optimal cost allocations, we then allocate each sources or supply according to some points of demand (destinations). Assignment Method is a specialized form of optimization linear programming model that attempts to assign limited capacity to various demand points in a way that minimizes costs. The special cases of transportation problem included degeneracy (a condition that occurs when the number of occupied squares in any solution is less than the number of rows plus the number of columns minus 1 in a transportation table), unbalanced problems, and multiple optimal 
solutions. At this opportunity, we would like to demonstrate the multiple optimal solutions. We will see how the Assignment method may be viewed as a special case of solving the transportation problem.

\section{Key Words}

Assignment method, VAM and MODI, Northwest Corner and Stepping-Stone.

\section{PENDAHULUAN}

Render, B., Stair, R.M., Hanna, M.E. (2010:368), the transportation algorithm is an iterative procedure in which a solution to a transportation problem is found and evaluated using a special procedure to determine whether the solution is optimal. If it is optimal, the process stops. If it is not optimal, a new solution is generated. This new solution is at least as good as the previous one, and it is usually better. This new solution is then evaluated, and if it is not optimal, another solution is generated. The process continues until the optimal solution is found.

Render, B., Stair, R.M., Hanna, M.E. (2009:457), Stevenson W.J. (2009:743), dan Taylor B.W. (2010:248,258), pemikiran dari tulisan atau artikel ini adalah berdasarkan teori bahwa problem transportasi dan problem assignment keduanya adalah termasuk kategori linear programming dan menggunakan teknis pemecahan secara linear programming pula. Ide dari tulisan atau artikel ini muncul pertama kali pada saat penulis memberikan kuliah manajemen sains beberapa waktu yang lalu, dan penulis mempunyai kesimpulan bahwa sebenarnya problem transportasi dengan multiple optimal solutions dapat juga diselesaikan dengan Assignment method, VAM and MODI dan Northwest Corner and Stepping-Stone. Pertama kali penulis mencoba menghitung hasilnya dengan Assignment method, lalu membandingkannya dengan VAM and MODI, dan NorthwestCorner and Stepping-Stone, dan setelah melakukan pengamatan beberapa kali dan juga melakukan tes penyelesaian dengan program komputer POM-QM for Windows ternyata memberikan hasil yang sama baik dilihat dari alokasinya maupun total biaya optimalnya, sehingga penulis berkesimpulan bahwa penyelesaian problem transportasi dengan 
multiple optimal solutions dapat juga diselesaikan dengan Assignment method, VAM and MODI dan Northwest Corner and Stepping-Stone.

Supranto, J. (2006:186,193), VAM (Vogel's Approximation Method), yang disingkat dengan VAM walaupun tidak selalu menghasilkan pemecahan optimum akan tetapi bias juga memberikan pemecahan yang optimal. VAM tidak menjamin suatu penyelesaian yang optimum, akan tetapi sangat berguna karena alasan berikut ini: (1) sering menghasilkan pemecahan optimum, (2) dapat menghasilkan penyelesaian yang mendekati optimal dengan usaha yang tidak banyak, sehingga dapat dipergunakan untuk melangkah menuju ke pemecahan yang optimal.

Render, B., Stair, R.M., Hanna, M.E. (2009:446), VAM method tackles the problem of finding a good initial solution by taking into account the costs associated with each route alternative. This is something that Northwest Corner Rule does not do. To apply VAM, we first compute for each row and column the penalty faced if we should ship over the second-best route instead of the least-cost route. After the initial VAM solution has been found, you should evaluate it with either the Stepping-Stone method or the MODI method. The MODI (Modified Distribution) method allows us to compute improvement indices quickly for each unused square without drawing all of the closed paths. Because of this, it can often provide considerable time savings over the SteppingStone method for solving transportation problems. If there is a negative index indicating an improvement can be made, then only one Stepping-Stone path must be found. This is used as it was before to determine what changes should be made to obtain the improved solution.

Render, B., Stair, R.M., Hanna, M.E. (2010:370, 372, 393), in the NorthwestCorner rule, the largest possible allocation is made to the cell in the upper left-hand corner of the tableau, followed by allocations to adjacent feasible cells. While the Stepping-Stone method is an iterative technique for moving from an initial feasible solution to an optimal feasible solution, and continues until the optimal solution is reached. The Stepping-Stone path method is used to calculate improvement indices for the empty cells. Improved solutions are developed using a Stepping-Stone path. 
Render, B., Stair, R.M., Hanna, M.E. (2010:385), each assignment problem has associated with a table, or matrix. Generally, the rows contain the objects or people we wish to assign and the columns comprise the tasks or things we want them assigned to. The numbers in the table are the cost associated with each particular assignment.

An assignment problem can be viewed as a transportation problem in which the capacity from each source (or person to be assigned) is 1 and the demand at each destination (or job to be done) is 1. Such formulation could be solved using the transportation algorithm, but it would have a severe degeneracy problem. However, this type of problem is very easy to solve using the assignment method.

Winston, W.L. (2004:393), although the transportation simplex appears to be very efficient, there is a certain class of transportation problems, called assignment problems, for which the transportation simplex is often very inefficient. We define assignment problems and discuss an efficient method that can be used to solve them.

Anderson, D.R., Sweeney, D.J., Williams, T.A., Martin, K. (2008:436), in generalizations of the assignment problem where one agent can be assigned to two or more tasks. Thus, we see that one advantage of formulating and solving assignment problems as linear programs is that special cases such as the situation involving multiple assignments can be easily handled.

Problem transportasi, dari tahun ke tahun perkuliahan biasanya diselesaikan dengan cara perhitungan VAM and MODI dan Northwest-Corner rule and Stepping-Stone, akan tetapi biasanya lupa atau tidak terdeteksi suatu problem dengan 2 (dua) atau lebih penyelesaian optimal (multiple optimal solutions) walaupun sudah menggunakan teori yang sangat rumit dan kompleks.

Pada kesempatan ini, penulis ingin menyampaikan penyelesaian multiple optimal solutions dengan menggunakan Assignment method, VAM and MODI dan Northwest Corner and Stepping-Stone. Yang dimaksud dengan multiple optimal solutions adalah penyelesaian suatu problem transportasi dengan hasil optimal yang lebih dari 1 (satu) jawaban, bisa 2 jawaban atau lebih, dan dengan total biaya minimal/optimal yang sama besarnya untuk tiap jawaban. 
Problem Transportasi:

\begin{tabular}{|l|l|l|l|l|}
\hline & \multicolumn{4}{|l|}{ Destination (Cost/unit) } \\
\hline Source & 1 & 2 & 3 & Supply \\
\hline A & $\$ 10$ & $\$ 4$ & $\$ 11$ & 70 \\
B & $\$ 12$ & $\$ 5$ & $\$ 8$ & 50 \\
C & $\$ 9$ & $\$ 7$ & $\$ 6$ & 30 \\
\hline Demand & 40 & 50 & 60 & $150 / 150$ \\
\hline
\end{tabular}

\section{Assignment Method:}

\begin{tabular}{|l|l|l|l|l|}
\hline 1 & \multicolumn{4}{|l|}{ Destination (Cost/unit) } \\
\hline Source & 1 & 2 & 3 & Supply \\
\hline A & 6 & 0 & 7 & 70 \\
B & 7 & 0 & 3 & 50 \\
C & 3 & 1 & 0 & 30 \\
\hline Demand & 40 & 50 & 60 & $150 / 150$ \\
\hline
\end{tabular}

\begin{tabular}{|l|l|l|l|l|}
\hline 2 & \multicolumn{4}{|l|}{ Destination (Cost/unit) } \\
\hline Source & 1 & 2 & 3 & Supply \\
\hline A & 3 & 0 & 7 & 70 \\
B & 4 & 0 & 3 & 50 \\
C & 0 & 1 & 0 & 30 \\
\hline Demand & 40 & 50 & 60 & $150 / 150$ \\
\hline
\end{tabular}

\begin{tabular}{|l|l|l|l|l|}
\hline 3 & \multicolumn{4}{|l|}{ Destination (Cost/unit) } \\
\hline Source & 1 & 2 & 3 & Supply \\
\hline A & 3 & $\uparrow$ & 7 & 70 \\
B & 4 & $\oint$ & 3 & 50 \\
C & 0 & & 0 & 30 \\
\hline Demand & 40 & 50 & 60 & $150 / 150$ \\
\hline
\end{tabular}




\begin{tabular}{|l|l|l|l|l|}
\hline 4 & \multicolumn{4}{|l|}{ Destination (Cost/unit) } \\
\hline Source & 1 & 2 & 3 & Supply \\
\hline A & 0 & 0 & 4 & 70 \\
B & 1 & 0 & 0 & 50 \\
C & 0 & 4 & 0 & 30 \\
\hline Demand & 40 & 50 & 60 & $150 / 150$ \\
\hline
\end{tabular}

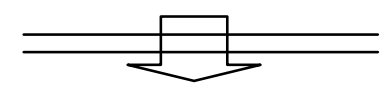

\section{Option 1}

\begin{tabular}{|l|l|l|l|l|}
\hline 5 & \multicolumn{4}{|l|}{ Destination (Cost/unit) } \\
\hline Source & 1 & 2 & 3 & Supply \\
\hline A & 0 & 0 & 4 & 70 \\
B & 1 & 0 & 0 & 50 \\
C & 0 & 4 & 0 & 30 \\
\hline Demand & 40 & 50 & 60 & $150 / 150$ \\
\hline
\end{tabular}

if we cannot find one zero at any row or column, find two zeros at any row or column instead.

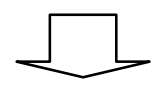

\begin{tabular}{|l|l|l|l|l|}
\hline Option 1 & \multicolumn{4}{|l|}{ Destination (Cost/unit) } \\
\hline Source & 1 & 2 & 3 & Supply \\
\hline A & 40 & 30 & & 70 \\
B & & 20 & 30 & 50 \\
C & & & 30 & 30 \\
\hline Demand & 40 & 50 & 60 & $150 / 150$ \\
\hline
\end{tabular}




\begin{tabular}{|l|}
\hline Total Minimal Cost Option 1 \\
\hline $\mathrm{A} 1=40 \times \$ 10=\$ 400$ \\
$\mathrm{~A} 2=30 \times \$ 4=\$ 120$ \\
$\mathrm{~B} 2=20 \times \$ 5=\$ 100$ \\
$\mathrm{~B} 3=30 \times \$ 8=\$ 240$ \\
$\mathrm{C} 3=30 \times \$ 6=\$ 180$ \\
\hline Total Cost Option 1=\$1,040 \\
\hline
\end{tabular}

\section{Option 2}

\begin{tabular}{|l|l|l|l|l|}
\hline 6 & \multicolumn{4}{|l|}{ Destination (Cost/unit) } \\
\hline Source & 1 & 2 & 3 & Supply \\
\hline A & 0 & 0 & 4 & 70 \\
B & 1 & 0 & 0 & 50 \\
C & 0 & 4 & 0 & 30 \\
\hline Demand & 40 & 50 & 60 & $150 / 150$ \\
\hline
\end{tabular}

if we cannot find one zero at any row or column, find

two zeros at any row or column instead.

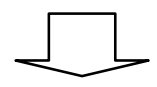

\begin{tabular}{|l|l|l|l|l|}
\hline Option 2 & \multicolumn{4}{|l|}{ Destination (Cost/unit) } \\
\hline Source & 1 & 2 & 3 & Supply \\
\hline A & 20 & 50 & & 70 \\
B & & & 50 & 50 \\
C & 20 & & 10 & 30 \\
\hline Demand & 40 & 50 & 60 & $150 / 150$ \\
\cline { 2 - 5 } & &
\end{tabular}




\begin{tabular}{|l|}
\hline Total Minimal Cost Option 2 \\
\hline $\mathrm{A} 1=20 \times \$ 10=\$ 200$ \\
$\mathrm{~A} 2=50 \times \$ 4=\$ 200$ \\
$\mathrm{~B} 3=50 \times \$ 8=\$ 400$ \\
$\mathrm{C} 1=20 \times \$ 9=\$ 180$ \\
$\mathrm{C} 3=10 \times \$ 6=\$ 60$ \\
\hline Total Cost Option $2=\mathbf{\$ 1 , 0 4 0}$ \\
\hline
\end{tabular}

\section{VAM and MODI:}

\section{VAM}

\begin{tabular}{|c|c|c|c|c|c|c|c|}
\hline 1 & \multicolumn{4}{|c|}{ Destination (Cost/unit) } & \multicolumn{3}{|c|}{ Opportunity Cost } \\
\hline Source & 1 & 2 & 3 & Supply & 1 & 2 & 3 \\
\hline A & $\$ 10$ & $\$ 4$ & $\$ 11$ & 70 & 6 & 1 & 1 \\
\hline B & $\$ 12$ & $\$ 5$ & $\$ 8$ & 50 & 3 & 4 & \\
\hline $\mathrm{C}$ & $\$ 9$ & $\$ 7$ & $\$ 6$ & 30 & 1 & 3 & 3 \\
\hline Demand & 40 & 50 & 60 & $150 / 150$ & & & \\
\hline 1 & 3 & 1 & 2 & & & & \\
\hline 2 & 3 & - & 2 & & & & \\
\hline 3 & 3 & - & 5 & & & & \\
\hline
\end{tabular}




\section{MODI}

\begin{tabular}{|c|c|c|c|c|}
\hline 2 & Destin & ion $(\mathrm{Co}$ & unit) & \\
\hline Source & $1(\mathrm{~K} 1)$ & $2(\mathrm{~K} 2)$ & $3(\mathrm{~K} 3)$ & Supply \\
\hline $\mathrm{A}(\mathrm{R} 1)$ & 20 & 50 & & 70 \\
\hline $\mathrm{B}(\mathrm{R} 2)$ & & & 50 & 50 \\
\hline C(R3) & 20 & & 10 & 30 \\
\hline Demand & 40 & 50 & 60 & $150 / 150$ \\
\hline $\mathrm{R} 1+\mathrm{K} 1=$ & 10, letti & $\mathrm{R} 1=0$, & $1=10$ & \\
\hline $\mathrm{R} 1+\mathrm{K} 2=$ & $4,0+K$ & $=4, \mathrm{~K} 2$ & & \\
\hline $\mathrm{R} 3+\mathrm{K} 1=$ & 9, R3 + & $=9, \mathrm{R} 3$ & & \\
\hline $\mathrm{R} 3+\mathrm{K} 3=$ & $6,-1+\mathrm{H}$ & $=6, \mathrm{~K} 3$ & & \\
\hline $\mathrm{R} 2+\mathrm{K} 3=$ & $8, \mathrm{R} 2+$ & $=8, \mathrm{R} 2$ & & \\
\hline Improvem & ent Index & $\mathrm{iij}=\mathrm{Cij}$ & $\mathrm{Ri}-\mathrm{Kj}$ & \\
\hline $\mathrm{I} 13=\mathrm{C} 13$ & - R1 - K & $=11-0$ & $=+4$ & \\
\hline $\mathrm{I} 21=\mathrm{C} 21$ & - R2 - K & $=12-1$ & $0=+1$ & \\
\hline $\mathrm{I} 22=\mathrm{C} 22$ & - R2 - K & $=5-1-$ & $=0$ & \\
\hline $\mathrm{I} 32=\mathrm{C} 32$ & - R3 - K & $=7-(-1)$ & $4=+4$ & \\
\hline
\end{tabular}

\section{Option 1}

\begin{tabular}{|l|l|l|l|l|}
\hline 3 & \multicolumn{4}{|l|}{ Destination (Cost/unit) } \\
\hline Source & 1 & 2 & 3 & Supply \\
\hline A & 20 & 50 & & 70 \\
B & & & 50 & 50 \\
C & 20 & & 10 & 30 \\
\hline Demand & 40 & 50 & 60 & $150 / 150$ \\
\hline
\end{tabular}




\begin{tabular}{|l|}
\hline Total Minimal Cost Option 1 \\
\hline $\mathrm{A} 1=20 \times \$ 10=\$ 200$ \\
$\mathrm{~A} 2=50 \times \$ 4=\$ 200$ \\
$\mathrm{~B} 3=50 \times \$ 8=\$ 400$ \\
$\mathrm{C} 1=20 \times \$ 9=\$ 180$ \\
$\mathrm{C} 3=10 \times \$ 6=\$ 60$ \\
\hline Total Cost Option $1=\mathbf{\$ 1 , 0 4 0}$ \\
\hline
\end{tabular}

Karena terdapat Improvement Index $\mathrm{I} 22=0$, mengindikasikan bahwa terdapat penyelesaian optimal yang lebih dari satu (multiple optimal solutions). Menggunakan stepping-stone closed path yang dimulai dari I22, maka angka terkecil di tiap sel stepping-stone closed path adalah 20, oleh karena itu 20 akan dikurangkan pada setiap sel dengan tanda negatif, dan 20 akan ditambahkan pada setiap sel dengan tanda positif.

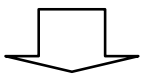

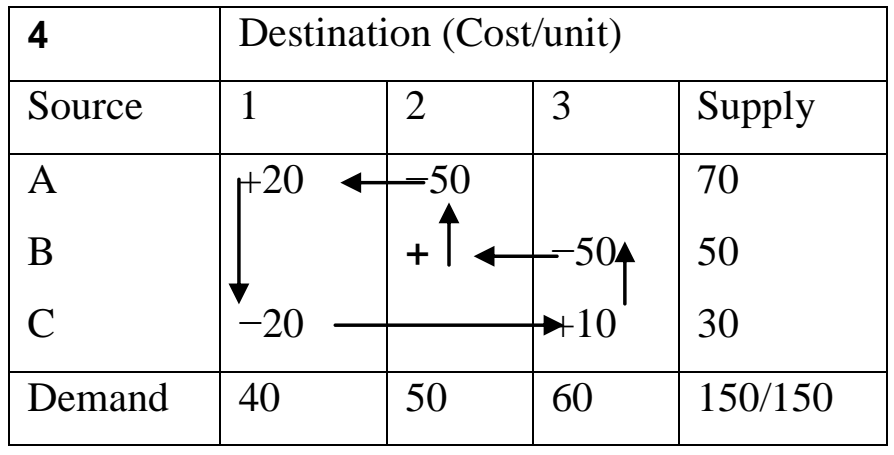

\section{Option 2}

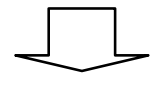

\begin{tabular}{|l|l|l|l|l|}
\hline 5 & \multicolumn{3}{|l|}{ Destination (Cost/unit) } \\
\hline Source & 1 & 2 & 3 & Supply \\
\hline A & 40 & 30 & & 70 \\
B & & 20 & 30 & 50 \\
C & & & 30 & 30 \\
\hline Demand & 40 & 50 & 60 & $150 / 150$ \\
\hline
\end{tabular}




\begin{tabular}{|l|}
\hline Total Minimal Cost Option 2 \\
\hline $\mathrm{A} 1=40 \times \$ 10=\$ 400$ \\
$\mathrm{~A} 2=30 \times \$ 4=\$ 120$ \\
$\mathrm{~B} 2=20 \times \$ 5=\$ 100$ \\
$\mathrm{~B} 3=30 \times \$ 8=\$ 240$ \\
$\mathrm{C} 3=30 \times \$ 6=\$ 180$ \\
\hline Total Cost Option $2=\mathbf{\$ 1 , 0 4 0}$ \\
\hline
\end{tabular}

\section{Northwest Corner and Stepping-Stone:}

\section{Northwest Corner}

\begin{tabular}{|l|l|l|l|l|}
\hline & \multicolumn{4}{|l|}{ Destination (Cost/unit) } \\
\hline Source & 1 & 2 & 3 & Supply \\
\hline A & $\$ 10$ & $\$ 4$ & $\$ 11$ & 70 \\
B & $\$ 12$ & $\$ 5$ & $\$ 8$ & 50 \\
C & $\$ 9$ & $\$ 7$ & $\$ 6$ & 30 \\
\hline Demand & 40 & 50 & 60 & $150 / 150$ \\
\hline
\end{tabular}

\begin{tabular}{|l|l|l|l|l|}
\hline $\mathbf{1}$ & \multicolumn{4}{|l|}{ Destination (Cost/unit) } \\
\hline Source & 1 & 2 & 3 & Supply \\
\hline A & 40 & 30 & & 70 \\
B & & 20 & 30 & 50 \\
C & & & 30 & 30 \\
\hline Demand & 40 & 50 & 60 & $150 / 150$ \\
\hline
\end{tabular}




\section{Stepping-Stone}

\begin{tabular}{|l|l|l|l|l|}
\hline 2 & \multicolumn{3}{|l|}{ Destination (Cost/unit) } \\
\hline Source & 1 & 2 & 3 & Supply \\
\hline A & 40 & 30 & & 70 \\
B & & 20 & 30 & 50 \\
C & & & 30 & 30 \\
\hline Demand & 40 & 50 & 60 & $150 / 150$ \\
\hline
\end{tabular}

$$
\begin{aligned}
& \text { Improvement } \\
& \text { Index: } \\
& \mathrm{I} 13=+\mathrm{A} 3-\mathrm{A} 2+\mathrm{B} 2-\mathrm{B} 3=+11-4+5-8=+4 \\
& \mathrm{I} 21=+\mathrm{B} 1-\mathrm{B} 2+\mathrm{A} 2-\mathrm{A} 1=+12-5+4-10=+1 \\
& \mathrm{I} 31=+\mathrm{C} 1-\mathrm{C} 3+\mathrm{B} 3-\mathrm{B} 2+\mathrm{A} 2-\mathrm{A} 1=+9-6+8-5+4-10=0 \\
& \mathrm{I} 32=+\mathrm{C} 2-\mathrm{C} 3+\mathrm{B} 3-\mathrm{B} 2=+7-6+8-5=+4
\end{aligned}
$$

\begin{tabular}{|c|c|c|c|c|}
\hline 3 & \multicolumn{4}{|c|}{ Destination (Cost/unit) } \\
\hline Source & 1 & 2 & 3 & Supply \\
\hline A & 40 & 30 & & 70 \\
\hline B & & 20 & 30 & 50 \\
\hline $\mathrm{C}$ & & & 30 & 30 \\
\hline Demand & 40 & 50 & 60 & $150 / 150$ \\
\hline
\end{tabular}

\section{Option 1}

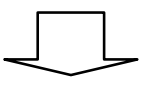

\begin{tabular}{|l|}
\hline Total Minimal Cost Option 1 \\
\hline $\mathrm{A} 1=40 \times \$ 10=\$ 400$ \\
$\mathrm{~A} 2=30 \times \$ 4=\$ 120$ \\
$\mathrm{~B} 2=20 \times \$ 5=\$ 100$ \\
$\mathrm{~B} 3=30 \times \$ 8=\$ 240$ \\
$\mathrm{C} 3=30 \times \$ 6=\$ 180$ \\
\hline Total Cost Option $1=\mathbf{\$ 1 , 0 4 0}$ \\
\hline
\end{tabular}


Karena terdapat Improvement Index $\mathrm{I} 31=0$, mengindikasikan bahwa terdapat penyelesaian optimal yang lebih dari satu (multiple optimal solutions). Menggunakan stepping-stone closed path yang dimulai dari I31, maka angka terkecil di tiap sel stepping-stone closed path adalah 20, oleh karena itu 20 akan dikurangkan pada setiap sel dengan tanda negatif, dan 20 akan ditambahkan pada setiap sel dengan tanda positif.

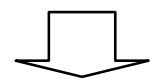

\begin{tabular}{|l|l|l|l|l|}
\hline 4 & \multicolumn{3}{|l|}{ Destination (Cost/unit) } \\
\hline Source & 1 & 2 & 3 & Supply \\
\hline A & -40 & -30 & & 70 \\
B & + & $-20 \longleftarrow+30$ & 50 \\
C & ++30 & & 30 \\
\hline Demand & 40 & 50 & 60 & $150 / 150$ \\
\hline
\end{tabular}

Option 2

\begin{tabular}{|l|l|l|l|l|}
\hline 5 & \multicolumn{3}{|l|}{ Destina tion (Cost/unit) } \\
\hline Source & 1 & 2 & 3 & Supply \\
\hline A & 20 & 50 & & 70 \\
B & & & 50 & 50 \\
C & 20 & & 10 & 30 \\
\hline Demand & 40 & 50 & 60 & $150 / 150$ \\
\cline { 5 - 5 } & &
\end{tabular}

\begin{tabular}{|l|}
\hline Total Minimal Cost Option 2 \\
\hline $\mathrm{A} 1=20 \times \$ 10=\$ 200$ \\
$\mathrm{~A} 2=50 \times \$ 4=\$ 200$ \\
$\mathrm{~B} 3=50 \times \$ 8=\$ 400$ \\
$\mathrm{C} 1=20 \times \$ 9=\$ 180$ \\
$\mathrm{C} 3=10 \times \$ 6=\$ 60$ \\
\hline Total Cost Option $2=\mathbf{\$ 1 , 0 4 0}$ \\
\hline
\end{tabular}




\section{KESIMPULAN}

Terdapat dua hasil optimal dalam penyelesaian problem transportasi di atas dengan Assignment method, VAM and MODI, Northwest Corner rule and Stepping-Stone method, dengan hasil alokasi yang berbeda akan tetapi menghasilkan total biaya minimal yang sama besarnya baik untuk alokasi yang terbentuk pada option 1 maupun untuk alokasi yang terbentuk pada option 2 .

The special cases of transportation problem, i.e. multiple optimal solutions, dapat diselesaikan dengan Assignment method, VAM and MODI, Northwest Corner rule and Stepping-Stone method, dan cara yang termudah adalah dengan Assignment method.

\section{DAFTAR PUSTAKA}

Anderson, D.R., Sweeney, D.J., Williams, T.A., Martin, K., 2008. An Introduction to Management Science, Quantitative Approaches to Decision Making (12 ${ }^{\text {th }}$ ed.). South-Western, a division of Thomson Learning, Mason, Ohio.

Anderson, D.R., Sweeney, D.J., Williams, T.A., Martin, K., 2008. Quantitative Methods for Business (11 thed.). South-Western, a part of The Thomson Corporation, Mason, Ohio.

Balakrishnan, N., Render, B., Stair, R.M., 2007. Managerial Decision Modeling with Spreadsheets ( $2^{\text {nd }}$ ed.). Pearson Education, Inc., Upper Saddle River, New Jersey.

Heizer, J., Render, B., 2011. Operations Management (10 ${ }^{\text {th }}$ ed.). Pearson Education, Inc., Upper Saddle River, New Jersey.

Hillier, F.S. et al., 2008. Introduction to Management Science, A Modelling and Case Studies Approach with Spreadsheets ( $3^{\text {rd }}$ ed.). McGraw-Hill/Irwin, New York.

Krajewski, L.J., Ritzman, L.P., Malhotra, M.K., 2010. Operations Management: Process and Value Chains ( $9^{\text {th }}$ ed.). Pearson Education, Inc., Upper Saddle River, New Jersey.

Render, B., Stair, R.M., Hanna, M.E., 2009. Quantitative Analysis for Management (10 ${ }^{\text {th }}$ ed.). Pearson Education, Inc., Upper Saddle River, New Jersey. 
Render, B., Stair, R.M., Hanna, M.E., 2012. Quantitative Analysis for Management (1 $1^{\text {th }}$ ed.). Pearson Education, Inc., Upper Saddle River, New Jersey.

Russell, R.S., Taylor, B.W., 2011. Operations Management ( $7^{\text {th }}$ ed.). John Wiley \& Sons (Asia) Pte, Ltd, Singapore.

Stevenson, W.J., 2009. Operations Management (10 ${ }^{\text {th }}$ ed.). McGraw-Hill/Irwin, New York.

Stevenson, W.J., Ozgur, C., 2007. Introduction to Management Science with Spreadsheets. McGraw-Hill/Irwin, New York.

Supranto, J., 2006. Riset Operasi Untuk Pengambilan Keputusan (2 ${ }^{\text {nd }}$ ed., edisi revisi). Penerbit Universitas Indonesia, Jakarta.

Taha, H.A., 2007. Operations Research: An Introduction ( $8^{\text {th }}$ ed.). Pearson Education, Inc., Upper Saddle River, New Jersey.

Taylor, B.W., 2010. Introduction to Management Science $\left(10^{\text {th }}\right.$ ed.). Pearson Education, Inc., Upper Saddle River, New Jersey.

Winston, W.L., 2004. Operations Research Application and Algorithms (4 ${ }^{\text {th }}$ ed.) edition. Brooks/Cole, a division of Thomson Learning, Inc., California. 